\title{
RADIO-AGN IN THE AKARI-NEP FIELD AND THEIR ROLE IN THE EVOLUTION OF GALAXIES
}

\author{
M. Karouzos, M. Im, And the Akari-NeP Team \\ Department of Physics and Astrnomy, Seoul National University, Seoul 151-747, Korea \\ Center for the Exploration of the Origin of the Universe \\ E-mail:mkarouzos@astro.snu.ac.kr \\ (Received June 28, 2012; Accepted July 27, 2012)
}

\begin{abstract}
Radio-loud active galaxies have been found to exhibit a close connection to galactic mergers and host galaxy star-formation quenching. We present preliminary results of an optical spectroscopic investigation of the AKARI NEP field. We focus on the population of radio-loud AGN and use photometric and spectroscopic information to study both their star-formation and nuclear activity components. Preliminary results show that radio-AGN are associated with early type, massive galaxies with relatively old stellar populations.
\end{abstract}

Key words: infrared: telescope; conferences: proceedings

\section{INTRODUCTION}

In the context of galaxy evolution in the Universe, the role of nuclear activity, in particular radio-loud active galactic nuclei (AGN), is still under debate. Are radioAGN a phase of a galaxy's evolution? How are they triggered and what is their effect on their host galaxy? We identify radio-AGN within the AKARI-NEP field and study their host galaxy properties in terms of an hierarchical evolutionary scheme.

\section{CROSS-IDENTIFICATION}

We cross-identify all AKARI-NEP (wide and deep) sources detected in the N2 band of AKARI with the sources from the WSRT catalog at $1.5 \mathrm{GHz}$ ([WH10]), following [DO86] (also see [WH12]). In total 401 and 168 radio sources are matched for NEP-wide and -deep, respectively. Photo-z for NEP-deep cross matched sources range between 0.37 and 2.2 , with most sources having $\mathrm{z}$ between 0.37 and 1 .

We also cross-identify $1.5 \mathrm{GHz}$ WSRT sources with the optical spectroscopy catalogs available (Shim et al., Takagi et al., private communication). For a matching radius of 3 arcsec, 48 radio sources are matched (spec-z between 0.03 and 4 , with a few above 1 ). Radio-samples are defined in Table 1.
TABLE 1.

Radio Samples Selection Criteria

\begin{tabular}{ccc}
\hline \hline Sample & Description & Selection \\
\hline$(1)$ & All & - \\
$(2)$ & Luminosity & $L_{1.5 G H z}>10^{23} \mathrm{~W} / \mathrm{Hz}^{*}$ \\
$(3)$ & Flat-spectrum & $\alpha_{\text {radio }}<0.5^{* *}$ \\
\hline \multirow{2}{*}{ *limit definition following [CO92][MA07] } \\
${ }^{* *} \alpha_{\text {radio }}$ calculated using 2 or 3 bands \\
\hline
\end{tabular}

\section{OPTICAL SPECTROSCOPY}

We are in the process of analyzing all the available optical spectra using IDL routines (emission line fluxes, equivalent widths, 4,000 $\AA$ break, etc.). Using the BPT emission line classification diagram (Fig. 1) we find $10 \mathrm{AGN} /$ LINERs and 21 transitional objects in a total of 84 sources. 3 radio-sources are classified as AGN/LINERs and 3 as transitional.

\section{OPTICAL COLORS AND STELLAR AGES}

We find that luminosity-selected AGN are predominantly associated with elliptical galaxies (ur $>2.22$; [ST01]), with a similar trend for our flatspectrum sample. A two-sample KS test gives a $99.8 \%$ 


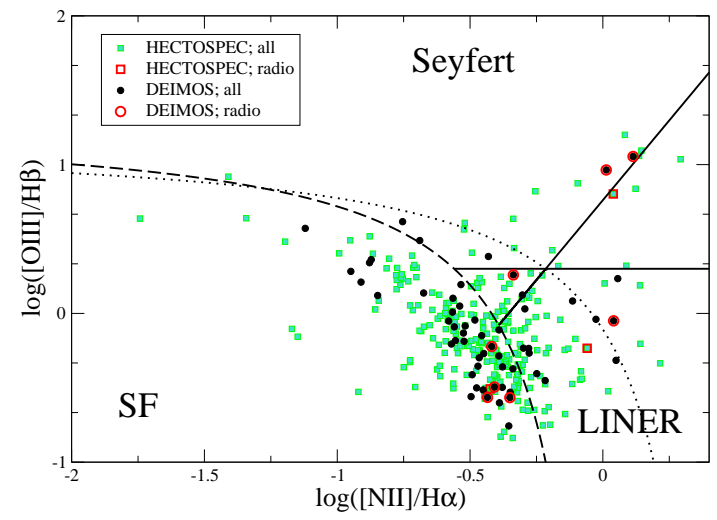

Fig. 1. Baldwin-Phillips-Terlevich (BPT) emission line ratios diagnostic diagram to separate star-forming galaxies from active AGN and LINERs. The dashed line is from [n], the dotted line from [l], and the continuous lines are from [m] (horizontal) and [n] (diagonal). They separate star-forming galaxies, LINERs, and Seyferts, respectively.

probability that sample (2) is drawn from a different population than its parent sample. For the comparison with sample (3) the KS test does not provide a significant result. Both samples (1) and (2) show $C_{4000 \AA}$ characteristic of old stellar populations and early-type galaxies (e.g.,[GA05]). A small fraction of sample (2) shows low values of C4000 indicative of a strong powerlaw non-thermal continuum (Fig. 2). Assuming that rest-frame N2 luminosity is a good proxy for the stellar mass of a galaxy, both samples (1) and (2) inhabit more massive galaxies compared to the non-radio sample.

\section{ACKNOWLEDGEMENTS}

MK acknowledges the support from the Creative Research Initiative program, No. 2010-0000712, of the National Research Foundation of Korea (NRFK) funded by the Korea government(MEST).

\section{REFERENCES}

Condon, J. J., 1992, Radio Emission from Normal Galaxies, ARA\&A, 30, 575

Downes, A. J., Peacock, J. A., Savage, A., et al., 1986, The Parkes Selected Regions - Powerful Radio Galaxies and Quasars at High Redshifts, MNRAS, 218, 31

Gallazzi, A., Charlot, S., Brinchmann, J., et al., 2005,
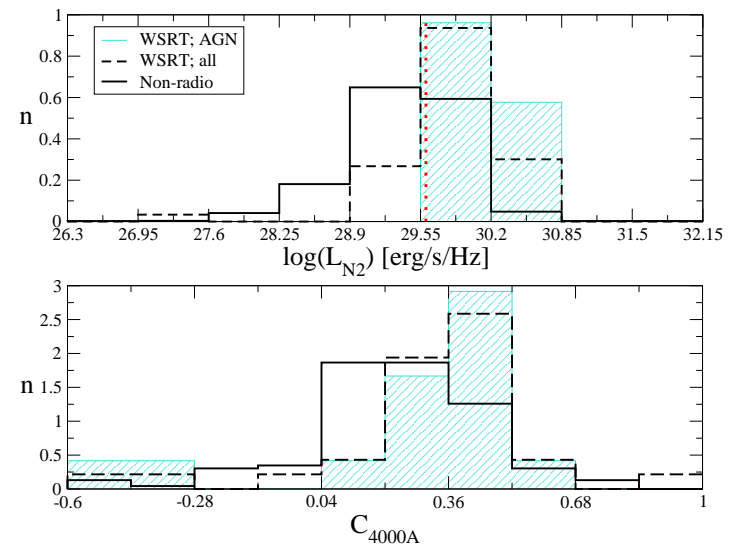

Fig. 2. Normalized distributions of the $4000 \mathrm{~A}$ break index C4000A and rest-frame N2 luminosity for all non-radio sources (black), all radio sources (dashed black), and luminosity-selected radio-AGN (shaded turquoise). Only sources with spectroscopic redshifts are included here. The dotted line denotes the $\mathrm{L}_{*}$.

The Ages and Metallicities of Galaxies in the Local Universe, MNRAS, 362, 41

Ho, L. C., Fillippenko, A. V., \& Sargent, W. L, 1997, A Search for "Dwarf" Seyfert Nuclei. III. Spectroscopic Parameters and Properties of the Host Galaxies, ApJS, 112, 315

Kauffmann, G., Heckman, T. M., Tremonti, C., et al., 2003, The Host Galaxies of Active Galactic Nuclei, MNRAS, 346, 1055

Kewley, L. J. \& Dopita, M. A., 2002, Using Strong Lines to Estimate Abundances in Extragalactic H II Regions and Starburst Galaxies, ApJS, 142, 35

Mauch, T. \& Sadler, E. M., 2007, Radio Sources in the 6dFGS: Local Luminosity Functions at $1.4 \mathrm{GHz}$ for Star-Forming Galaxies and Radio-Loud AGN, MNRAS, 375, 931

Strateva, I., Ivezić, Ż., Knapp, G. R., et al., 2001, Color Separation of Galaxy Types in the Sloan Digital Sky Survey Imaging Dat, AJ, 122, 1861

White, G. J., Pearson, C., Braun, R., et al., 2010, A Deep Survey of the AKARI North Ecliptic Pole Field. I. WSRT $20 \mathrm{~cm}$ Radio Survey Description, Observations and Data Reduction, A\&A, 517A, 54

White, G. J., Hatsukade, B., Pearson, C., et al., 2012, A deep ATCA $20 \mathrm{~cm}$ Radio Survey of the AKARI Deep Field South near the South Ecliptic Pole, arXiv:1207.2262 